\title{
Consensus Analysis for a Class of Heterogeneous Multiagent Systems with Time Delay Based on Frequency Domain Method
}

\author{
Yi-Jie Sun, Guo-liang Zhang, and Jing Zeng \\ High-Tech Institute of Xian, Xian, Shanxi 710025, China \\ Correspondence should be addressed to Yi-Jie Sun; syjlxh@126.com
}

Received 4 May 2014; Accepted 17 August 2014; Published 28 September 2014

Academic Editor: Housheng Su

Copyright ( 2014 Yi-Jie Sun et al. This is an open access article distributed under the Creative Commons Attribution License, which permits unrestricted use, distribution, and reproduction in any medium, provided the original work is properly cited.

\begin{abstract}
The consensus problem of heterogeneous multiagent systems composed of first-order and second-order agent is investigated. A linear consensus protocol is proposed. Based on frequency domain method, the sufficient conditions of achieving consensus are obtained. If communication topology contains spanning tree and some conditions can be satisfied on control gains, consensus can be achieved. Then, a linear consensus protocol with time delay is proposed. In this case, consensus is dependent only on system coupling strength, each agent input time delay, but independent of communication delay. Finally, numerical simulations are provided to illustrate the effectiveness of the theoretical result.
\end{abstract}

\section{Introduction}

Recently, multiagent systems have received significant attention due to their potential impact on numerous civilians, homeland securities, and military applications. Consensus plays an important role in achieving distributed coordination. The basic idea of consensus is that a team of agents reach an agreement on a common value by negotiating with their neighbors. Many literatures have investigated consensus problem on many cases, such as time variant topology, time delay, and nonlinearity [1-10].

Unfortunately, all the aforementioned multiagent systems are homogeneous; that is, all the agents share the same dynamics behavior. However, the dynamics of the agents are quite different because of various restrictions in the practical systems. Zheng et al. [11] studied the consensus problem of heterogeneous multiagent systems composed of first-order and second-order integrator agents. Zhu et al. [12] studied consensus problem of multiagent systems with two types of agents, namely, active agents and passive agents. Yin et al. [13] investigated the consensus problem for a set of discrete-time heterogeneous multiagent systems composed of two kinds of agents differed by their dynamics. Zhu et al. [14] investigated the finite-time consensus problem for heterogeneous multiagent systems composed of first-order and second-order agents. Zheng and Wang [15] studied finitetime consensus of heterogeneous multiagent systems with and without velocity measurements. C. Liu and F. Liu [16] considered stationary consensus of heterogeneous multiagent systems with bounded communication delays. Yan et al. [17] are concerned with the cooperative target pursuit problem by multiple agents based on directed acyclic graph. Kim et al. [18] studied the output consensus problem for a class of heterogeneous uncertain linear multiagent systems. Yin et al. [19] studied the consensus protocols design for a set of fractional-order heterogeneous agents, which is composed of two kinds of agents. Kim et al. [20] investigated the heterogeneous consensus problem for multiagent systems with random link failures between agents.

Consensus of heterogeneous multiagent systems is investigated in undirected graph generally. An important challenge is to study this problem in directed graph. However, this seems to be less studied in the literature. Meanwhile, frequency domain approach is an important method for analyzing stability of control system. In the existing literatures, there are so few literatures that use frequency domain method. Time delay appears in almost all practical systems, and times delay can degrade the systems performance or even destroy the system stability. 
In this paper, we study consensus problem of heterogeneous multiagent systems with and without time delay. The model and algorithms in this paper are similar to [11], but in [11] they only consider the case in undirected graph mainly based on Lyapunov theory. Unlike [11], in this paper consider the cases with and without time delay in directed graph. Based on the frequency domain method, the sufficient conditions are given for the existence of consensus solution to heterogeneous multiagent systems. By these conditions, it is shown that consensus can be achieved when control gains satisfy some conditions in the case without time delay. In the case with time delay, the results show that the consensus is dependent only on system coupling strength, each agent input time delay, but independent of communication delay.

The rest of the paper is organized as follows. In Section 2, some preliminaries are introduced for the graph theory, and heterogeneous multiagent systems are formulated. Two consensus protocols are proposed in Section 3, and consensus analysis is shown for without delay time and with delay time, respectively. In Section 4, two numerical examples are studied. Finally, concluding remarks are given in Section 5.

\section{Preliminaries}

2.1. Graph Theory. Let $\mathscr{G}(\mathscr{V}, \mathscr{E}, \mathscr{A})$ be a weighted directed graph composed of a set of nodes $\mathscr{V}=\left\{v_{1}, v_{2}, \ldots, v_{n}\right\}$, set of edges $\mathscr{E} \subset \mathscr{V} \times \mathscr{V}$, and a weighted adjacency matrix $A=\left[a_{i j}\right]_{n \times n}$, with nonnegative adjacency elements $a_{i j}$. An edge of $\mathscr{G}$ is denoted by $e_{i j}=\left(v_{i}, v_{j}\right) \in \mathscr{E}$, which means that node $v_{i}$ receives information from node $v_{j}$ and $v_{j}$ is called the parent of $v_{i}$. The adjacency elements associated with the edges of the graph are positive; that is, $e_{i j} \in \mathscr{E} \Leftrightarrow a_{i j}>0$. Moreover, we assume $a_{i i}=0$ for all $i \in 1, \ldots, n$. The set of neighbors of node $v_{i}$ is denoted by $N_{i}=\left\{v_{j} \in \mathscr{V}:\left(v_{i}, v_{j}\right) \in \mathscr{E}\right\}$. The corresponding graph Laplacian $L=\left[l_{i j}\right]_{n \times n}$ can be defined as

$$
l_{i j}= \begin{cases}-a_{i j}, & j \neq i, \\ \sum_{j \in N_{i}} a_{i j}, & j=i .\end{cases}
$$

If $a_{i j}=a_{j i}$, then $\mathscr{G}$ becomes the undirected graph. If there is not isolated node in an undirected graph, the graph is said to be connected.

Graph with a Spanning Tree. For a directed graph, if every node, except a node called root, has exactly one parent, then it is called a directed tree. A spanning tree of a directed graph is a directed tree formed by graph edges that connect all the nodes of the graph.

In heterogeneous multiagent system, the neighbors of each second-order agent $i$ include first-order and secondorder agents, denoted by $N_{i}=N_{i}^{s} \cup N_{i}^{f}$, and the neighbors of each first-order agent are denoted by $N_{l}=N_{l}^{s} \cup N_{l}^{f}$. The Laplacian matrix can be denoted as follows:

$$
L=\left[\begin{array}{cc}
\bar{L}_{s} & -A_{s f} \\
-A_{f s} & \bar{L}_{f}
\end{array}\right],
$$

where $\bar{L}_{s}=D_{s f}+L_{s}, L_{s}$ is the Laplacian matrix of secondorder agents, $D_{s f}=\operatorname{diag}\left(\sum_{j \in N_{i}^{f}} a_{i j}, i=1, \ldots, m\right)$, and $A_{s f}$ denotes the adjacency relations of second-order agent to firstorder agent. Meanwhile, $L_{f}$ is the Laplacian matrix of firstorder agent, $D_{f_{s}}=\operatorname{diag}\left(\sum_{j \in N_{l}^{s}} a_{l j}, l=m+1, \ldots, n\right)$, and $A_{f_{s}}$ denotes the adjacency relations of first-order agents to second-order agents.

2.2. The Heterogeneous Multiagent System. Suppose that the heterogeneous multiagent system consists of first-order and second-order integrator agents. The number of agents is $n$, labeled from 1 through $n$. Firstly, the heterogeneous multiagent system and concept of consensus are established. Suppose that the number of second-order integrator agents is $m(m<n)$. The remainder is the first-order integrator agents; the number is $n-m$. Then, the system is given as follows:

$$
\begin{gathered}
\dot{x}_{i}=v_{i}, \\
\dot{v}_{i}=u_{i}, \quad i=1, \ldots, m, \\
\dot{x}_{l}=u_{l}, \quad l=m+1, \ldots, n,
\end{gathered}
$$

where $x_{i}, v_{i}, u_{i}$ are the position, velocity, and control input, of second-order agent $i$, respectively. $x_{l}, u_{l}$ are the position and control input of first-order agent $l$, respectively. The initial conditions are $\mathbf{x}(0)=\left[x_{1}(0), x_{2}(0), \ldots, x_{n}(0)\right]^{T}$ and $\mathbf{v}(0)=$ $\left[v_{1}(0), v_{2}(0), \ldots, v_{m}(0)\right]^{T}$.

Definition 1. The heterogeneous multiagent system (3) is said to reach consensus asymptotically if, for any initial conditions $\mathbf{x}(0)$ and $\mathbf{v}(0)$, one has $\lim _{t \rightarrow \infty}\left\|x_{j}(t)-x_{i}(t)\right\|=0, i, j=$ $1, \ldots, n, \lim _{t \rightarrow \infty}\left\|v_{j}(t)-v_{i}(t)\right\|=0, i, j=1, \ldots, m$.

Lemma 2 (see [5]). Suppose that $\mathbf{z}=\left[z_{1}, z_{2}, \ldots, z_{p}\right]^{T}$ with $z_{i} \in R$ and $L \in R^{p \times p}$ is the Laplacian matrix. Then, the following four conditions are equivalent: (i) L has a simple zero eigenvalue with an associated eigenvector $\mathbf{1}_{p}$ and all of the other eigenvalues have positive real parts; (ii) $L z=0$ implies that $z_{1}=z_{2} \cdots=z_{p}$; (iii) consensus is reached asymptotically for a system $z=-L z$; (iv) the directed graph of $L$ has a directed spanning tree.

Remark 3. Throughout the paper, we just take the positions and velocities of agents as scalars. However, all the following developments can be directly extended to the case of vectors by introducing the Kronecker product.

\section{Main Results}

3.1. Consensus Protocol without Time Delay. In this section, the protocol without time delay is proposed for system (3), as follows:

$$
\begin{aligned}
& u_{i}=\sum_{j \in N_{i}} a_{i j}\left(x_{j}-x_{i}\right)-k_{1} v_{i}, \quad i=1, \ldots, m, \\
& u_{l}=k_{2} \sum_{j \in N_{l}} a_{i j}\left(x_{j}-x_{l}\right), \quad l=m+1, \ldots, n,
\end{aligned}
$$

where $k_{1}, k_{2}>0$ are control gains. 
Theorem 4. If the following conditions can hold, then consensus of system (3) with protocol (4) can be achieved.

(i) The fixed topology contains spanning tree. (ii) The control gains satisfy conditions

$$
k_{1} \geq \sqrt{2 \max \left\{d_{i}, i=1, \ldots, m\right\}}, \quad k_{2}>0 .
$$

Proof. The system (3) under protocol (4) can be written in a vector form as

$$
\dot{z}(t)=\Lambda z(t),
$$

where

$$
\Lambda=\left[\begin{array}{ccc}
0 & I_{m} & 0 \\
-\left(L_{s}+D_{s f}\right) & -k_{1} I_{m} & A_{s f} \\
k_{2} A_{f s} & 0 & -k_{2}\left(L_{f}+D_{f s}\right)
\end{array}\right]
$$

where $\mathbf{z}(t)=\left[x_{1}(t), \ldots, x_{m}(t), v_{1}(t), \ldots, v_{m}(t), x_{m+1}(t), \ldots\right.$, $\left.x_{n}(t)\right]^{\mathrm{T}}$.

The Laplace transformation is imposed on system (6) and we have

$$
\begin{gathered}
s^{2} x_{s}(s)=-\left(L_{s}+D_{s f}\right) x_{s}(s)-k_{1} s x_{s}(s)+A_{s f} x_{f}(s), \\
s x_{f}(s)=-k_{2}\left(L_{f}+D_{f s}\right) x_{f}(s)+k_{2} A_{f_{s}} x_{s}(s) .
\end{gathered}
$$

From (8), the following form is obtained:

$$
\begin{aligned}
& s x_{s}(s)=\frac{-\left(L_{s}+D_{s f}\right) x_{s}(s)-s^{2} x_{s}(s)+A_{s f} x_{f}(s)}{k_{1}}, \\
& s x_{f}(s)=-\left(L_{f}+D_{f s}\right) x_{f}(s)+A_{f_{s}} x_{s}(s) .
\end{aligned}
$$

Let $x_{s}=\left[x_{1}, \ldots, x_{m}\right]^{\mathrm{T}}, x_{f}=\left[x_{m+1}, \ldots, x_{n}\right]^{\mathrm{T}}$, and $y=$ $\left[x_{s}^{\mathrm{T}}, x_{f}^{\mathrm{T}}\right]^{\mathrm{T}}$; we have

$$
\begin{gathered}
s y(s)=\Gamma(s) y(s) \\
\Gamma(s)=\left[\begin{array}{cc}
\frac{-\left(L_{s}+D_{s f}\right)-s^{2} I}{k_{1}} & \frac{A_{s f}}{k_{1}} \\
k_{2} A_{f s} & -k_{2}\left(L_{f}+D_{f s}\right)
\end{array}\right] .
\end{gathered}
$$

Let $F(s)=\operatorname{det}(s I-\Gamma(s))$. It is the fact that the zero point of $F(s)$ is the eigenvalue of $\Lambda$. Consider the following.

(1) When $s=0, F(0)=\operatorname{det}(\Gamma(0))=$ $\left(1 / k_{1}\right)^{m}\left(k_{2}\right)^{n-m} \operatorname{det}(L)$. Zero is a simple eigenvalue of $L$ owing to the topology that contains spanning tree based on Lemma 2. Then, $F(0)=0$ and the zero point is $s=0$.

(2) When $s \neq 0$, let $P(s)=\operatorname{det}(I+G(s)), G(s)=$ $-\Gamma(s) / s$. Based on the generalized Nyquist criterion, if the point $(-1,0 j)$ is not encircled by Nyquist curve of $G(s)$, then zero points of $P(s)$ are all with negative real part.
Let $s=j \omega$, according to Gershgorin disk theorem, the eigenvalues $\lambda(G(j \omega))$ of matrix $G(j \omega)$ are all in below circles. One can see that

$$
\begin{gathered}
\lambda(G(j \omega)) \in\left\{G_{i}, i=1, \ldots, m\right\} \bigcup\left\{G_{l}, l=m+1, \ldots, n\right\}, \\
G_{i}=\left\{\xi: \xi \in C|| \xi-\frac{\sum_{j \in N_{i}} a_{i j}}{j \omega k_{1}}-\frac{j \omega}{k_{1}}\left|\leq \sum_{j \in N_{i}}\right| \frac{a_{i j}}{j \omega k_{1}} \mid\right\}, \\
i=1, \ldots, m, \\
G_{l}=\left\{\xi: \xi \in C|| \xi-\frac{k_{2} \sum_{j \in N_{i}} a_{i j}}{j \omega}\left|\leq \sum_{j \in N_{i}}\right| \frac{k_{2} a_{i j}}{j \omega} \mid\right\}, \\
l=m+1, \ldots, n,
\end{gathered}
$$

where $G_{i}, i=1, \ldots, m$, are considered firstly. Let $d_{i}=$ $\sum_{j \in N_{i}} a_{i j}$, and the center of circle $G_{i}$ is $G_{i 0}(j \omega)=\left(\left(\omega / k_{1}\right)-\right.$ $\left.\left(d_{i} / \omega k_{1}\right)\right) j$.

If the point $(-a, 0 j), a \geq 1$, is not in $G_{i}$, then

$$
\left|-a-\frac{d_{i}}{j \omega k_{1}}-\frac{j \omega}{k_{1}}\right| \geq \sum_{j \in N_{i}}\left|\frac{a_{i j}}{j \omega k_{1}}\right| .
$$

Then, we have

$$
2 d_{i}-\omega^{2} \leq k_{1}^{2}
$$

It is easy to see that if $k_{1} \geq \sqrt{2 \max \left\{d_{i}, i=1, \ldots, m\right\}}$, then (20) can be established.

Therefore, $k_{1} \geq \sqrt{2 \max \left\{d_{i}, i=1, \ldots, m\right\}}$, and the point $(-1,0 j)$ is not encircled by $G_{i}, i=1, \ldots, m$.

Next, $G_{l}, l=m+1, \ldots, n$, will be considered. If the point $(-a, 0 j), a \geq 1$, is not in $G_{l}$, then

$$
\left|-a-\frac{k_{2} d_{l}}{j \omega}\right| \geq \sum_{j \in N_{l}}\left|\frac{k_{2} a_{i j}}{j \omega}\right| .
$$

It is easy to verify that if $k_{2}>0,(14)$ can be established.

From the above analysis, we can get that matrix $\Lambda$ only has a simple eigenvalue and all nonzero eigenvalues with negative real part. Then, system (6) can converge a stable state, $\lim _{t \rightarrow \infty} \mathbf{z}(t)=\mathbf{z}^{*}(t)$, and $\Lambda \mathbf{z}^{*}(t)=0$, where $\mathbf{z}^{*}(t)=$ $\left[x_{1}^{*}(t), \ldots, x_{m}^{*}(t), v_{1}^{*}(t), \ldots, v_{m}^{*}(t), x_{m+1}^{*}(t), \ldots, x_{n}^{*}(t)\right]^{\mathrm{T}}$. It is easy to verify that the vector $c\left[\mathbf{1}_{m}^{\mathrm{T}}, \mathbf{0}_{m}^{\mathrm{T}}, \mathbf{1}_{n-m}^{\mathrm{T}}\right]^{\mathrm{T}}$ is eigenvector of matrix $\Lambda$ associated with eigenvalue 0 . So, the solution of $\Lambda \mathbf{y}^{*}(t)=0$ is $c\left[\mathbf{1}_{m}^{\mathrm{T}}, \mathbf{0}_{m}^{\mathrm{T}}, \mathbf{1}_{n-m}^{\mathrm{T}}\right]^{\mathrm{T}}$, where $c$ is a constant. We can get that $\lim _{t \rightarrow \infty} x_{i}(t)=c, i=1, \ldots, n, \lim _{t \rightarrow \infty} v_{i}(t)=$ $0, i=1, \ldots, m$. Based on Definition 1 , the system achieves the consensus. It can be observed from Theorem 4 that the control gains $k_{1}, k_{2}$ play important roles in achieving consensus.

3.2. Consensus Protocol with Time Delay. In this section, the case with time delay is considered. A consensus protocol with time delay is proposed for system (3). The different 
communication time delay and identical input time delay among agents are considered in following protocol:

$$
\begin{gathered}
u_{i}=\sum_{j \in N_{i}} a_{i j}\left(x_{j}\left(t-\tau_{i j}\right)-x_{i}(t-\tau)\right)-k_{1} v_{i}(t-\tau), \\
i=1, \ldots, m, \\
u_{l}=k_{2} \sum_{j \in N_{l}} a_{i j}\left(x_{j}\left(t-\tau_{i j}\right)-x_{l}(t-\tau)\right), \quad l=m+1, \ldots, n,
\end{gathered}
$$

where $k_{1}, k_{2}>0$ are control gains, $\tau_{i j}$ is communication time delay, and $\tau$ is input time delay.

Theorem 5. If the following conditions can be hold, then consensus of system (3) with protocol (15) can be achieved.

(i) The fixed topology contains spanning tree. (ii) The following conditions can be established: $k_{1} \geq$ $\sqrt{2 \max \left\{d_{i}, i=1, \ldots, m\right\}}, k_{2}>0$, and $\tau \leq \min \left\{1 / 2 k_{1}\right.$, $\left.1 / 2 k_{2} \max \left\{d_{l}, l=m+1, \ldots, n\right\}\right\}$.

Proof. The Laplace transformation is imposed on system (3) with protocol (15), and we have

$$
\begin{gathered}
s x_{i}(s)=v_{i}(s), \\
s v_{i}(s)=\sum_{j \in N_{i}} a_{i j}\left(e^{-\tau_{i j} s} x_{j}(s)-e^{-\tau s} x_{i}(s)\right)-k_{1} e^{-\tau s} v_{i}(s), \\
i=1, \ldots, m, \\
s x_{l}(s)=\sum_{j \in N_{l}} a_{i j}\left(e^{-\tau_{i j} s} x_{j}(s)-e^{-\tau s} x_{l}(s)\right), \\
l=m+1, \ldots, n .
\end{gathered}
$$

Let $\widetilde{L}=\left[\widetilde{l}_{i j}\right]_{n \times n}$

$$
\tilde{l}_{i j}= \begin{cases}e^{-\tau_{i j s}} a_{i j}, & i \neq j, \\ \sum_{j \in N_{i}} a_{i j} e^{-\tau s}, & i=j .\end{cases}
$$

We have

$$
\begin{gathered}
s^{2} x_{s}(s)=-\left(\widetilde{L}_{s}+\widetilde{D}_{s f}\right) x_{s}(s)-k_{1} e^{-\tau s} s x_{s}(s)+\widetilde{A}_{s f} x_{f}(s) \\
s x_{f}(s)=-k_{2}\left(\widetilde{L}_{f}+\widetilde{D}_{f s}\right) x_{f}(s)+k_{2} \widetilde{A}_{f s} x_{s}(s)
\end{gathered}
$$

Let $x_{s}=\left[x_{1}, \ldots, x_{m}\right]^{\mathrm{T}}, x_{f}=\left[x_{m+1}, \ldots, x_{n}\right]^{\mathrm{T}}$, and $y=$ $\left[x_{s}^{\mathrm{T}}, x_{f}^{\mathrm{T}}\right]^{\mathrm{T}}$; we have

$$
\begin{gathered}
s y(s)=\widetilde{\Gamma} y(s), \\
\widetilde{\Gamma}(s)=\left[\begin{array}{cc}
\frac{-\left(\widetilde{L}_{s}+\widetilde{D}_{s f}\right)-s^{2} I}{k_{1} e^{-\tau s}} & \frac{\widetilde{A}_{s f}}{k_{1} e^{-\tau s}} \\
k_{2} \widetilde{A}_{f s} & -k_{2}\left(\widetilde{L}_{f}+\widetilde{D}_{f s}\right)
\end{array}\right] .
\end{gathered}
$$

Let $\widetilde{F}(s)=\operatorname{det}(s I-\widetilde{\Gamma}(s))$. Next, the zero point of $F(s)$ will be analyzed. Consider the following.

(1) When $s=0, \widetilde{F}(0)=\operatorname{det}(\widetilde{\Gamma}(0))=$ $\left(1 / k_{1}\right)^{m}\left(k_{2}\right)^{n-m} \operatorname{det}(L)$. Zero is a simple eigenvalue of $L$ owing to the topology that contains spanning tree based on Lemma 2. Then, $F(0)=0$ and the zero point is $s=0$.

(2) When $s \neq 0$, let $\widetilde{P}(s)=\operatorname{det}(I+\widetilde{G}(s)), \widetilde{G}(s)=$ $-\widetilde{\Gamma}(s) / s$. Based on the generalized Nyquist criterion, if the point $(-1,0 j)$ is not encircled by Nyquist curve of $\widetilde{G}(s)$, then zero points of $\widetilde{P}(s)$ are all with negative real part.

Let $s=j \omega$; according to Gershgorin disk theorem, the eigenvalues $\lambda(\widetilde{G}(j \omega))$ of matrix $\widetilde{G}(j \omega)$ are all in below circles. Consider

$$
\begin{gathered}
\lambda(\widetilde{G}(j \omega)) \in\left\{\widetilde{G}_{i}, i=1, \ldots, m\right\} \bigcup\left\{\widetilde{G}_{l}, l=m+1, \ldots, n\right\}, \\
\widetilde{G}_{i}=\left\{\xi: \xi \in C|| \xi-\frac{\sum_{j \in N_{i}} a_{i j}}{j \omega k_{1}}-\frac{j \omega e^{\tau \omega j}}{k_{1}} \mid\right. \\
\left.\leq \sum_{j \in N_{i}}\left|\frac{a_{i j} e^{-\left(\tau_{i j}-\tau\right) \omega j}}{j \omega k_{1}}\right|\right\}, \quad i=1, \ldots, m, \\
\widetilde{G}_{l}=\left\{\begin{array}{l}
\xi: \xi \in C|| \xi-\frac{\sum_{j \in N_{l}} a_{l j} e^{-\tau \omega j}}{j \omega} \mid \\
\left.\leq \sum_{j \in N_{l}}\left|\frac{a_{l j} e^{-\tau_{l j \omega j}}}{j \omega}\right|\right\}, \quad l=m+1, \ldots, n .
\end{array}\right.
\end{gathered}
$$

Firstly, $\widetilde{G}_{i}, i=1, \ldots, m$, are considered. Let $d_{i}=$ $\sum_{j \in N_{i}} a_{i j}$, and the center of circle is $\widetilde{G}_{i 0}=-\left((\omega \sin \omega \tau) / k_{1}\right)+$ $\left(\left((\omega \cos \omega \tau) / k_{1}\right)-\left(d_{i} / k_{1} \omega\right)\right) j$.

If the point $(-a, 0 j), a \geq 1$, is not in $\widetilde{G}_{i}$, then

$$
\left|-a-\frac{\sum_{j \in N_{i}} a_{i j}}{j \omega k_{1}}-\frac{j \omega e^{\tau \omega j}}{k_{1}}\right| \geq \sum_{j \in N_{i}}\left|\frac{a_{i j} e^{-\left(\tau_{i j}-\tau\right) \omega j}}{j \omega k_{1}}\right| .
$$

We have

$$
\begin{gathered}
1-2 \frac{\omega \sin \omega \tau}{k_{1}}+\frac{\omega^{2}}{k_{1}^{2}}-2 \frac{\cos \omega \tau d_{i}}{k_{1}^{2}} \geq 0, \\
\omega^{2}\left(1-2 k_{1} \tau \frac{\sin \omega \tau}{\omega \tau}\right)+k_{1}^{2}-2 \cos \omega \tau d_{i} \geq 0 .
\end{gathered}
$$

If the following inequalities can hold, then (22) will be established:

$$
\begin{gathered}
\left(1-2 k_{1} \tau \frac{\sin \omega \tau}{\omega \tau}\right) \geq 0, \\
k_{1}^{2}-2 \cos \omega \tau d_{i} \geq 0 .
\end{gathered}
$$


Note that $\sin x / x \leq 1$ can be established for all $x \in R$ and $-1 \leq \cos x \leq 1$. Therefore, if the following conditions are satisfied, then the point $(-1,0 j)$ is not encircled by Nyquist curve of $\widetilde{G}_{i}(j \omega), i=1, \ldots, m$ :

$$
\begin{gathered}
\tau \leq \frac{1}{2 k_{1}}, \\
k_{1} \geq \sqrt{2 \max \left\{d_{i}, i=1, \ldots, m\right\}} .
\end{gathered}
$$

Next, $\widetilde{G}_{l}, l=m+1, \ldots, n$, are considered. If the point $(-a, 0 j), a \geq 1$, is not in $\widetilde{G}_{l}, l=m+1, \ldots, n$, then

$$
\left|-a-\frac{k_{2} \sum_{j \in N_{l}} a_{l j} e^{-\tau \omega j}}{j \omega}\right| \geq \sum_{j \in N_{l}}\left|\frac{k_{2} a_{l j} e^{-\tau_{l j \omega j}}}{j \omega}\right| .
$$

We have

$$
\begin{gathered}
a^{2}-2 a k_{2} \frac{\sin \omega \tau d_{l}}{\omega} \geq 0, \\
2 k_{2} \frac{\tau \sin \omega \tau d_{l}}{\omega \tau} \leq 1 .
\end{gathered}
$$

It is obvious that $\sin x / x \leq 1$ can be established for all $x \in R$. So, (29) can be satisfied, and (28) will hold:

$$
\tau \leq \frac{1}{2 k_{2} \max \left\{d_{l}, l=m+1, \ldots, n\right\}} .
$$

Combining the above analysis, Theorem 5 is demonstrated.

Remark 6. From Theorem 5, it can be seen that consensus condition of system (3) with time delay only is dependent on input time delay of each agent, but is independent of communication time delay.

In Theorem 5, each agent with identical input time delay is assumed. From the above analysis, the conclusion with different input time delay among agents can also be deduced from the conclusion of Theorem 5. Consider

$$
\begin{gathered}
u_{i}=\sum_{j \in N_{i}} a_{i j}\left(x_{j}\left(t-\tau_{i j}\right)-x_{i}\left(t-\tau_{i}\right)\right)-k_{1} v_{i}\left(t-\tau_{i}\right), \\
i=1, \ldots, m, \\
u_{l}=k_{2} \sum_{j \in N_{l}} a_{i j}\left(x_{j}\left(t-\tau_{i j}\right)-x_{l}\left(t-\tau_{i}\right)\right), \\
l=m+1, \ldots, n .
\end{gathered}
$$

Corollary 7. If the following conditions can hold, then consensus of system (3) with protocol (30) can be achieved.

(i) The fixed topology contains spanning tree. (ii) The following conditions can be established: $k_{1} \geq$ $\sqrt{2 \max \left\{d_{i}, i=1, \ldots, m\right\}}, k_{2}>0$, and $\tau_{i} \leq 1 / 2 k_{1}, i=1, \ldots, m$, $\tau_{l} \leq 1 / 2 k_{2} d_{l}$, and $l=m+1, \ldots, n$.

From Corollary 7, the upper bound of each $\tau_{i}, i=$ $1, \ldots, n$, can be established.

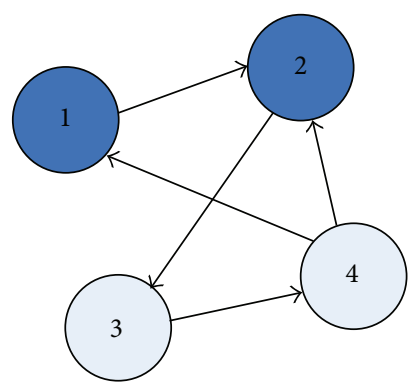

FIGURE 1: Interconnection graph.
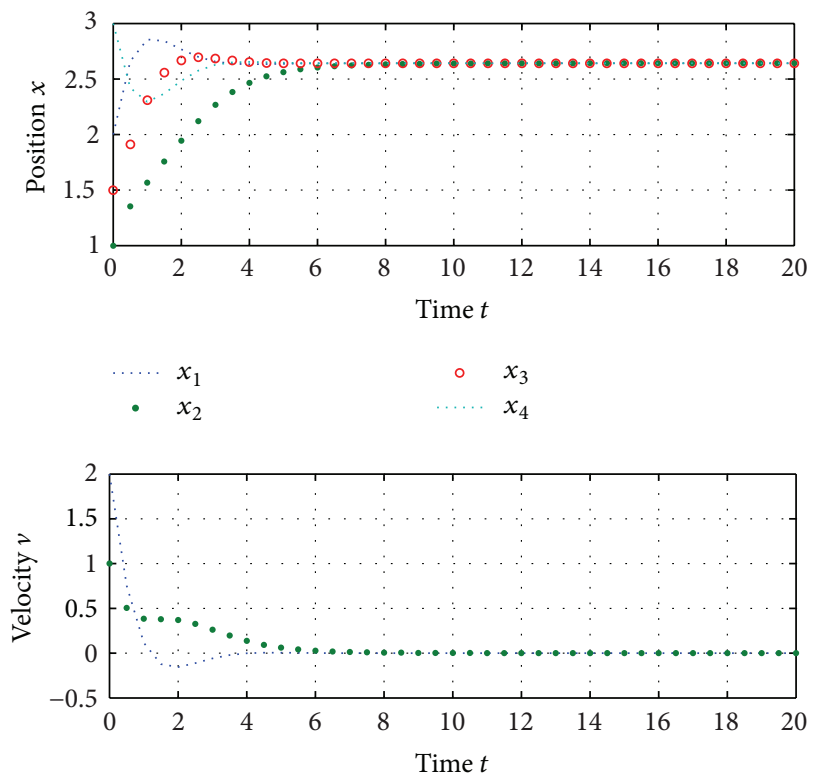

$\begin{array}{ll}\cdots \cdots & v_{1} \\ \text { - } & v_{2}\end{array}$

FIGURE 2: The state information of agents.

\section{Simulation}

In this section, several simulation results are presented to illustrate the proposed consensus algorithm introduced in Section 3.

Example 8. Consider a heterogeneous multiagent system with 4 agents, shown in Figure 1, where nodes 1, 2 are secondorder agents and nodes 3, 4 are first-order agents. The graph has a directed spanning tree apparently. If $e_{j i} \in \mathscr{E}$, then $a_{i j}=1$; else $a_{i j}=0$. The Laplacian matrix is given as follows:

$$
L=\left[\begin{array}{cccc}
1 & 0 & 0 & -1 \\
-1 & 2 & 0 & -1 \\
0 & -1 & 1 & 0 \\
0 & 0 & -1 & 1
\end{array}\right]
$$

According to the conditions in Theorem 4, we select $k_{1}=$ $2.1, k_{2}=1.2$. The initial conditions are $\mathbf{x}(0)=[2,1,1.5,3]^{\mathrm{T}}$, $\mathbf{v}(0)=[2,1]^{\mathrm{T}}$. The simulation results (Figure 2) show that 

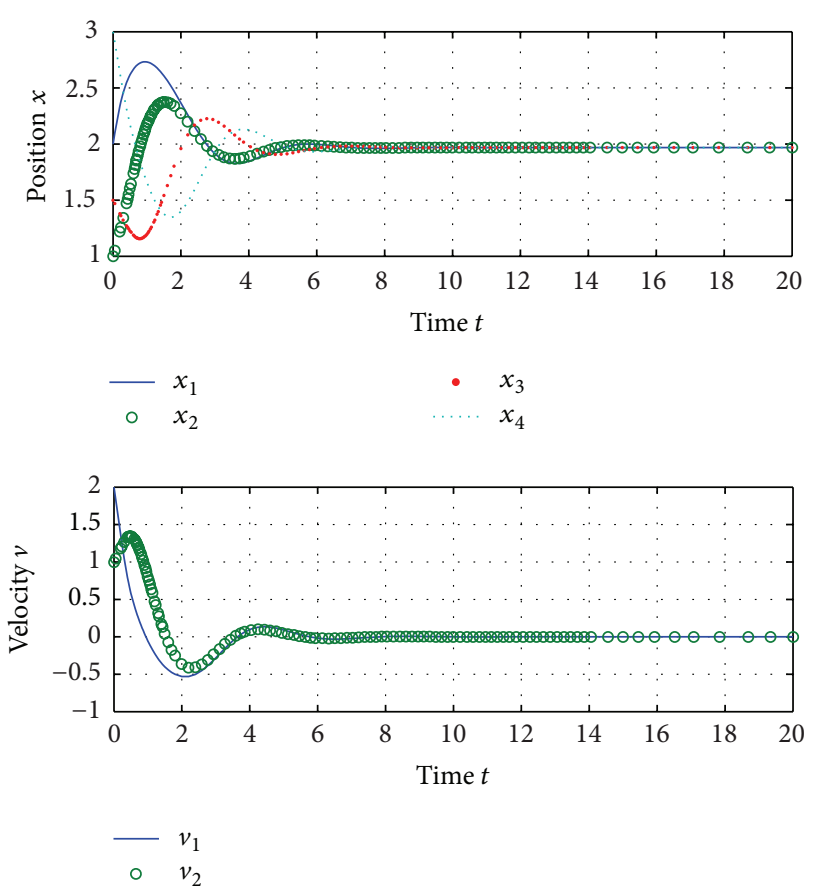

FIGURE 3: The state information of agents.
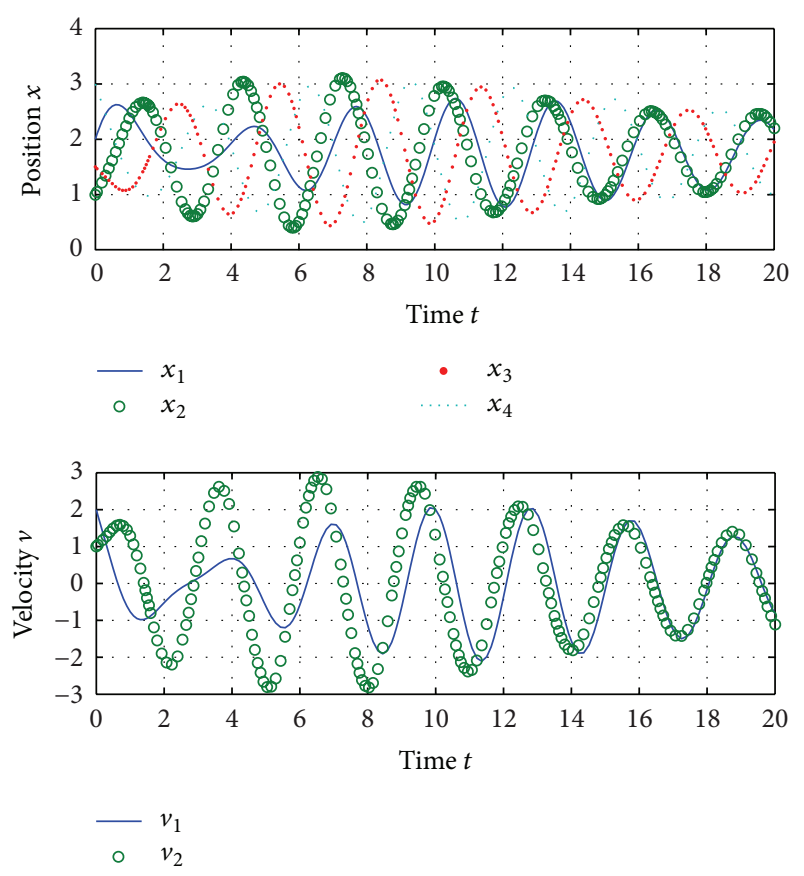

FIGURE 4: The state information of agents.

the positions of all agents and the velocities of second-order agents reach consensus asymptotically.

Example 9. These are the same communication topology and initial conditions as Example 8. The consensus protocol with time delay is considered. Firstly, control gains are selected as $k_{1}=2.1, k_{2}=1.2$. Based on the conditions in Theorem 5,
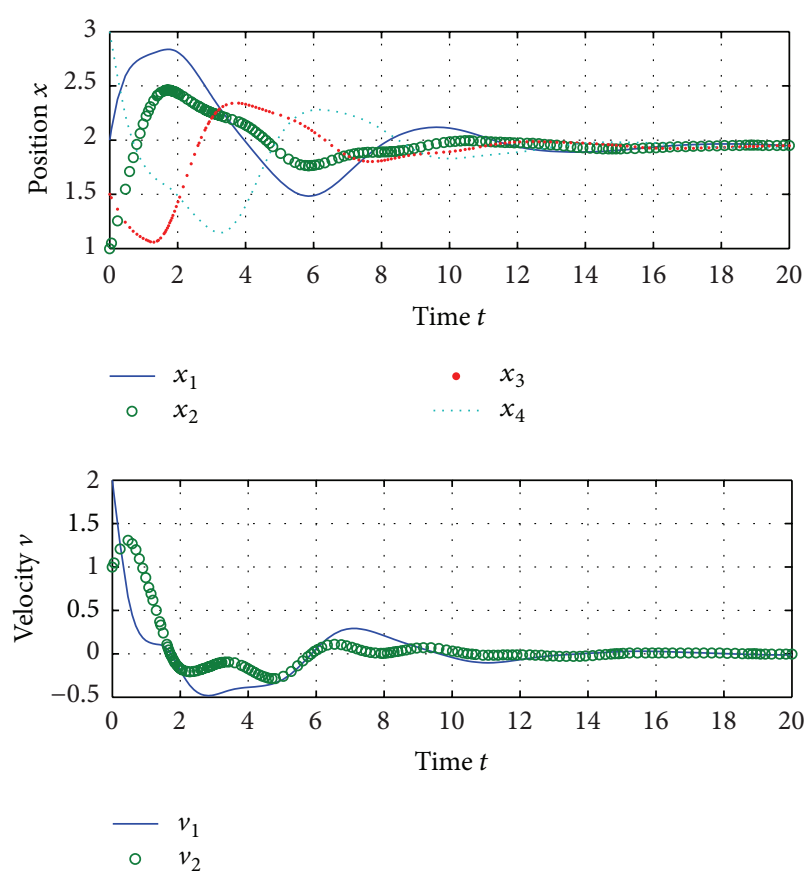

FIgURE 5: The state information of agents.

the upper bound of input time delay can be obtained and $\tau<0.2381$ can be derived. We selected $\tau=0.23$. The communication time delay among agents is set as $\tau_{12}=$ $0.2, \tau_{23}=0.6, \tau_{34}=0.4, \tau_{41}=0.5$, and $\tau_{42}=0.3$. The simulation results (Figure 3 ) show that the positions of all agents and the velocities of second-order agents reach consensus asymptotically. It can be seen that the speed of convergence of system with time delay is slower than the system without time delay.

Based on Example 9, input time delay is set as $\tau=0.5$, and the simulation result (Figure 4) shows that the system is divergent. So, consensus condition is dependent on input time delay.

Meanwhile, we selected $\tau=0.23$, and the communication time delays are enlarged as $\tau_{12}=1.5, \tau_{23}=1.2, \tau_{34}=1.6, \tau_{41}=$ 1.4 , and $\tau_{42}=1.2$. The simulation result (Figure 5 ) shows that the system is convergent. Consensus condition is dependent on input time delay. But, communication time delays can affect the speed of convergence of system obviously.

\section{Conclusion}

This paper focuses on the consensus problem of heterogeneous multiagent composed of first-order agent and second-order agent. The protocols with time delay and without time delay are investigated, respectively. The sufficient conditions of consensus are obtained in two cases. Consensus of the protocol without time delay is dependent on control gains and communication topology. If the control gains satisfy some conditions and communication topology contains spanning tree, then the consensus can be achieved. Consensus of the protocol with time delay is dependent only 
on system coupling strength, each agent input time delay, but independent of communication delay. Finally, numerical simulations are provided to illustrate the effectiveness of the theoretical result. From simulation, it can be seen that all communication time delays and input time delay can affect the speed of convergence of system obviously. In the future work, discrete-time heterogeneous with time delay will be considered.

\section{Conflict of Interests}

The authors declare that there is no conflict of interests regarding the publication of this paper.

\section{Acknowledgments}

The authors would like to thank the associate editor and the anonymous reviewers for their valuable comments and suggestions that have improved the presentation of this paper.

\section{References}

[1] T. Vicsek, A. Czirok, E. B. Jacob, I. Cohen, and O. Schochet, "Novel type of phase transition in a system of self-driven particles," Physical Review Letters, vol. 75, no. 6, pp. 1226-1229, 1995.

[2] A. Jadbabaie, J. Lin, and A. S. Morse, "Coordination of groups of mobile autonomous agents using nearest neighbor rules," IEEE Transactions on Automatic Control, vol. 48, no. 6, pp. 988-1001, 2003.

[3] R. Olfati-Saber and R. M. Murray, "Consensus problems in networks of agents with switching topology and time-delays," IEEE Transactions on Automatic Control, vol. 49, no. 9, pp. 15201533, 2004.

[4] W. Ren and R. W. Beard, "Consensus seeking in multi-agent systems under dynamically changing interaction topologies," IEEE Transactions on Automatic Control, vol. 50, no. 5, pp. 655661, 2005.

[5] L. Wang and F. Xiao, "A new approach to consensus problems in discrete-time multiagent systems with time-delays," Science in China F: Information Sciences, vol. 50, no. 4, pp. 625-635, 2007.

[6] F. Xiao and L. Wang, "State consensus for multi-agent systems with switching topologies and time-varying delays," International Journal of Control, vol. 79, no. 10, pp. 1277-1284, 2006.

[7] F. Xiao and L. Wang, "Asynchronous consensus in continuoustime multi-agent systems with switching topology and timevarying delays," IEEE Transactions on Automatic Control, vol. 53, no. 8, pp. 1804-1816, 2008.

[8] H. Su, X. Wang, and Z. Lin, "Flocking of multi-agents with a virtual leader," IEEE Transactions on Automatic Control, vol. 54, no. 2, pp. 293-307, 2009.

[9] H. Su, M. Z. Q. Chen, J. Lam, and Z. Lin, "Semi-global leaderfollowing consensus of linear multi-agent systems with input saturation via low gain feedback," IEEE Transactions on Circuits and Systems. I: Regular Papers, vol. 60, no. 7, pp. 1881-1889, 2013.

[10] H. Su, M. Z. Chen, X. Wang, and J. Lam, "Semiglobal observerbased leader-following consensus with input saturation," IEEE Transactions on Industrial Electronics, vol. 61, no. 6, pp. 28422850, 2014.
[11] Y. Zheng, Y. Zhu, and L. Wang, "Consensus of heterogeneous multi-agent systems," IET Control Theory \& Applications, vol. 5, no. 16, pp. 1881-1888, 2011.

[12] S. Zhu, C. Chen, and X. Guan, "Consensus protocol for heterogeneous multi-agent systems: a Markov chain approach," Chinese Physics B, vol. 22, no. 1, Article ID 018901, 2013.

[13] X. Yin, D. Yue, and S. Hu, "Distributed event-triggered control of discrete-time heterogeneous multi-agent systems," Journal of the Franklin Institute, vol. 350, no. 3, pp. 651-669, 2013.

[14] Y. Zhu, X. Guan, and X. Luo, "Finite-time consensus of heterogeneous multi-agent systems," Chinese Physics B, vol. 22, no. 3, Article ID 038901, 2013.

[15] Y. Zheng and L. Wang, "Finite-time consensus of heterogeneous multi-agent systems with and without velocity measurements," Systems \& Control Letters, vol. 61, no. 8, pp. 871-878, 2012.

[16] C. Liu and F. Liu, "Stationary consensus of heterogeneous multi-agent systems with bounded communication delays," Automatica, vol. 47, no. 9, pp. 2130-2133, 2011.

[17] J. Yan, X. Guan, and X. Luo, "Consensus pursuit of heterogeneous multi-agent systems under a directed acyclic graph," Chinese Physics B, vol. 20, no. 4, Article ID 048901, 2011.

[18] H. Kim, H. Shim, and J. H. Seo, "Output consensus of heterogeneous uncertain linear multi-agent systems," IEEE Transactions on Automatic Control, vol. 56, no. 1, pp. 200-206, 2011.

[19] X. Yin, D. Yue, and S. Hu, "Consensus of fractional-order heterogeneous multi-agent systems," IET Control Theory \& Applications, vol. 7, no. 2, pp. 314-322, 2013.

[20] J. M. Kim, J. B. Park, and Y. H. Choi, "Leaderless and leaderfollowing consensus for heterogeneous multi-agent systems with random link failures," IET Control Theory \& Applications, vol. 8, no. 1, pp. 51-60, 2014. 


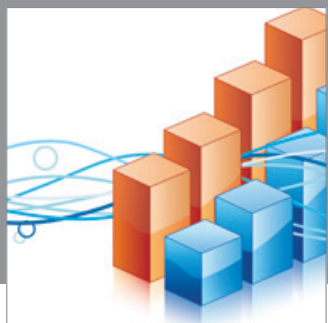

Advances in

Operations Research

mansans

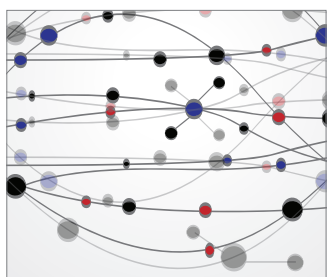

The Scientific World Journal
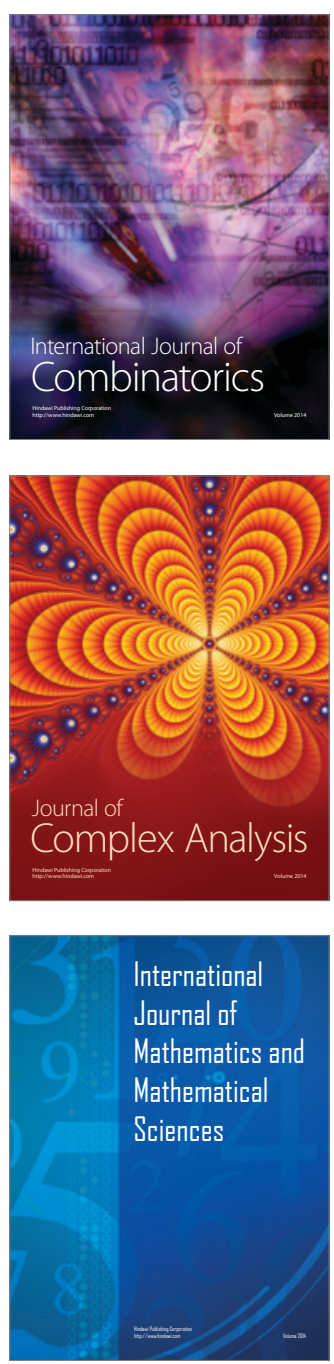
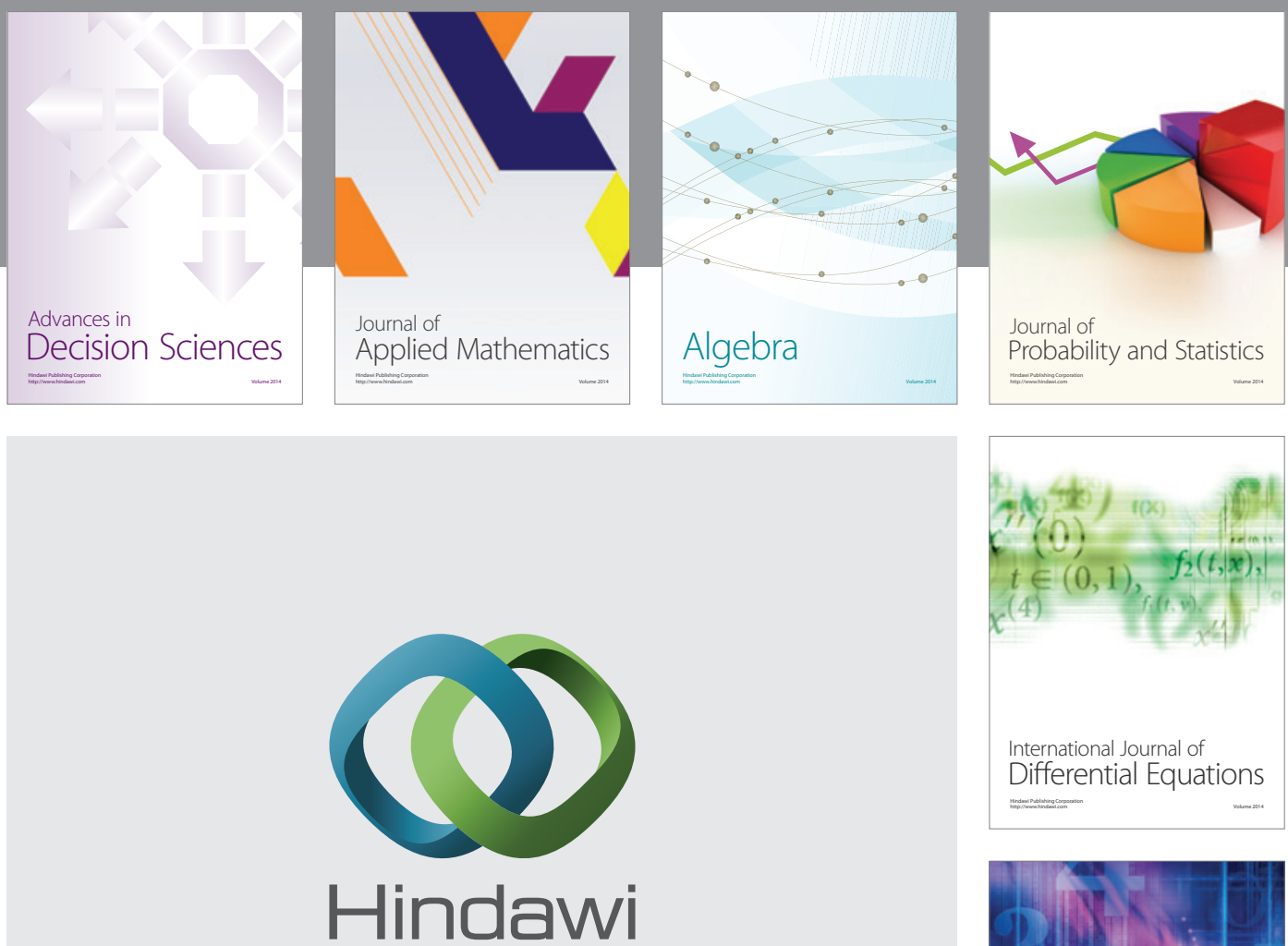

Submit your manuscripts at http://www.hindawi.com
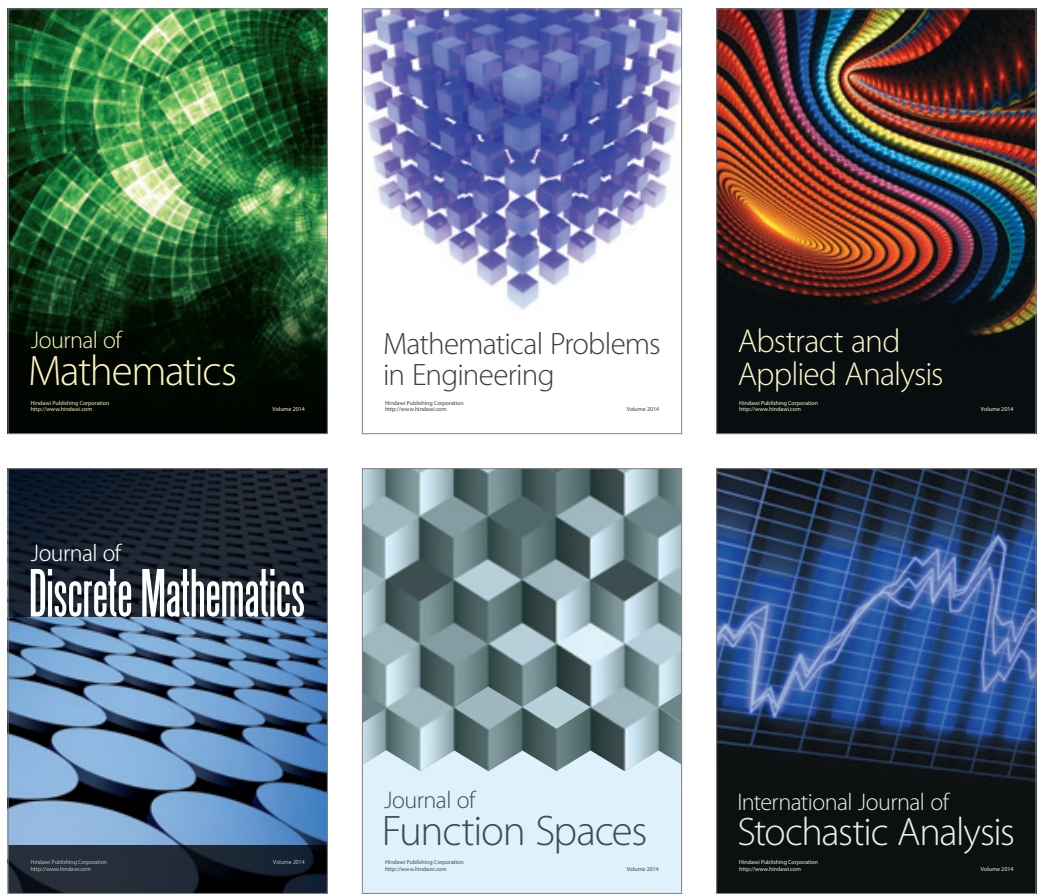

Journal of

Function Spaces

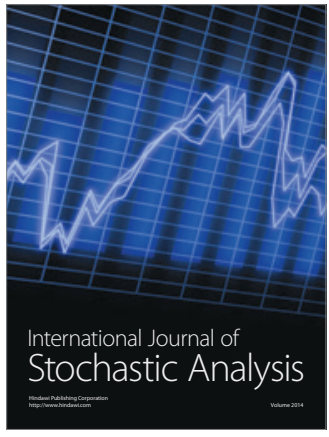

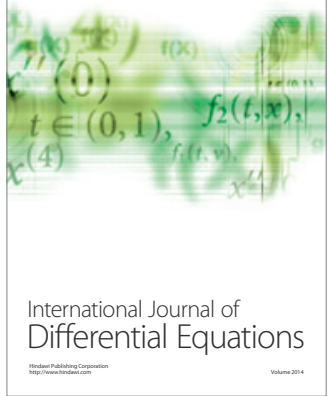
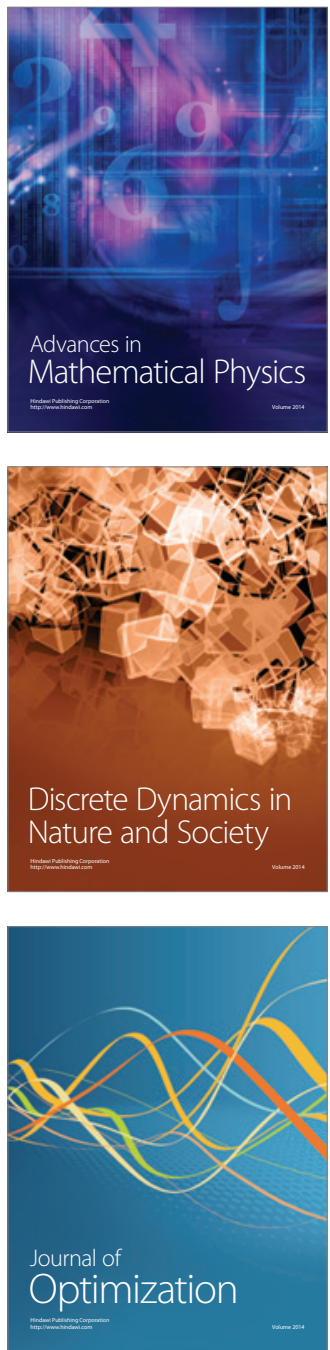